\title{
Isolation and characterisation of neurotoxigenic Clostridium butyricum from soil in China
}

\author{
X. MENG, K. YAMAKAWA*, K. ZOU, X. WANG, X. KUANG, C. LU, C. WANG, T. KARASAWA* and \\ S. NAKAMURA*
}

Department of Anaerobic Bacteriology, Lanzhou Institute of Biological Products, Ministry of Public Health of China, Lanzhou, China and *Department of Bacteriology, School of Medicine, Kanazawa University, Kanazawa 920-8640, Japan

\begin{abstract}
Soil specimens collected from a site around the home of patients with food-borne type $E$ botulism probably caused by neurotoxigenic Clostridium butyricum in Guanyun, Jiangsu province, China, were examined for the presence of neurotoxigenic $C$. butyricum. Five lakeside sites of Weishan lake, in an area near to the sites where the type $E$ botulism outbreaks caused by neurotoxigenic $C$. butyricum occurred were also surveyed. Type $\mathbf{E}$ toxin-producing $C$. butyricum was isolated from soil from four sites including the site in Guanyun. Polymerase chain reaction assay demonstrated the presence of the type $E$ toxin gene in all the toxigenic isolates. The biochemical properties of the isolates from the Guanyun soil and the lakeside soil were identical except for inulin fermentation and starch hydrolysis properties. These results indicate that neurotoxigenic $C$. butyricum has its principal habitat in soil.
\end{abstract}

\section{Introduction}

Clostridium botulinum is divided into seven toxigenic types $(A-G)$ on the basis of the serological specificity of the neurotoxins produced. It has been recognised that the neurotoxins are produced by four different clostridial groups based on cultural and biochemical properties or DNA-DNA homology [1]. However, in 1986, it was demonstrated that two clostridial species other than $C$. botulinum produced botulinum toxin in three cases of infant botulism, two in Rome, Italy [2, 3] and one in New Mexico [4]. Type E botulinum toxinproducing $C$. butyricum was isolated from the cases in Rome and type $\mathrm{F}$ botulinum toxin-producing $C$. baratii from the case in New Mexico.

Recently, food-borne botulism cases were encountered in Guanyun, Jiangsu province of China which may have been caused by type $\mathrm{E}$ botulinum toxin-producing $C$. butyricum. Clinical material was not examined in this outbreak, but the organism was isolated from the implicated food - salted and fermented paste made of soybeans and wax gourds $[5,6]$. This prompted the re-examination of the cultural and biochemical properties of type $\mathrm{E}$ botulinum toxinproducing strains which had been isolated from type $\mathrm{E}$

Received 6 Jan. 1998; revised version accepted 7 May 1998. Corresponding author: Professor S. Nakamura. botulism cases and kept in Lanzhou Institute of Biological Products. In this survey, two isolates from botulism cases that occurred in the Weishan lake area, in the eastern part of China, exhibited the properties of C. butyricum.

On the basis of these findings, soil from around the house of the patients involved in the botulism outbreak in Guanyun and from the Weishan lake area was examined for the presence of neurotoxigenic $C$. butyricum. The properties of the isolates obtained in this study, as well as two neurotoxigenic strains with the properties of $C$. butyricum found in a survey of strains from our collections, were examined in detail.

\section{Materials and methods \\ Collection strains tested}

Two stock strains with type E botulinum toxicity, which had been isolated at Lanzhou Institute from samples from food-borne botulism cases, were found, on reexamination, to have the properties of $C$. butyricum and were tested further for biochemical properties. One strain (LCL 063) had been isolated from the intestinal content of a botulism patient at Jining in 1973, and the other (LCL 095) from food implicated in botulism at Peixian in 1983 (Fig. 1). Neurotoxigenic C. butyricum BL 6340, which had been isolated in Rome [2], was also tested. 


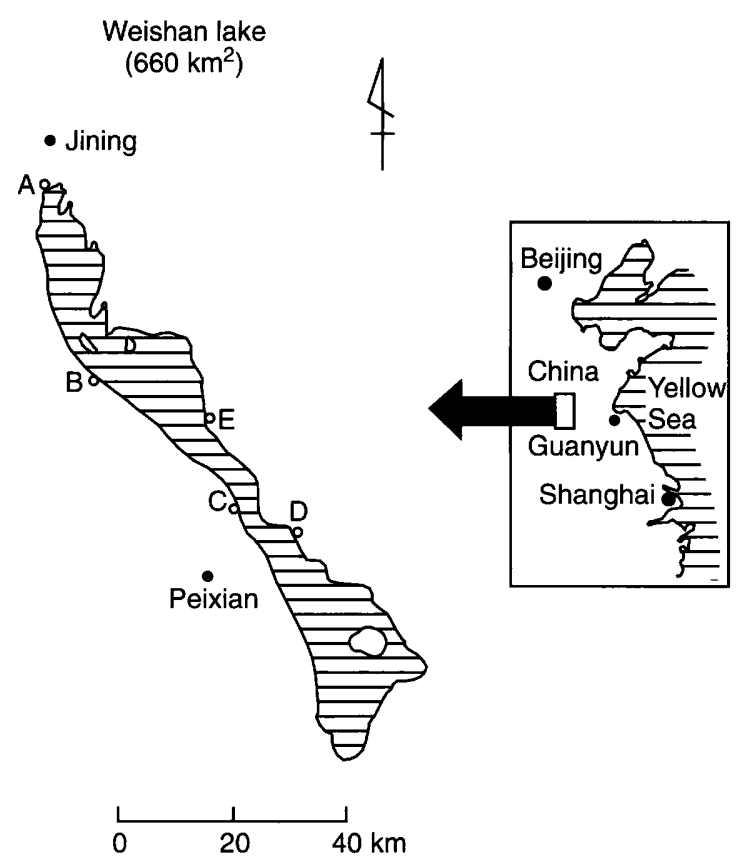

Fig. 1. Location of Guanyun and Weishan lake area. Soil specimens in the Weishan lake area were collected at sites A, B, C, D and E. Neurotoxigenic C. butyricum strains had been isolated in the botulism outbreak at Jining in 1973 and at Peixian in 1983.

\section{Soil specimens}

Soil specimens were collected in Guanyun, Jiangsu province, China, in June 1995, from sites around the house of the patients who had type E botulism in 1994 [6]; neurotoxigenic $C$. butyricum had been isolated from the implicated food [5]. Guanyun is about $180 \mathrm{~km}$ from Weishan lake. At Guanyun, soil specimens were taken from 10 different places in the cultivated fields at 100-m intervals extending out from the garden next to the patients' house.

In June 1995, soil specimens were collected from five sites on the shore of Weishan lake; all the sites were cultivated fields except for site A, which was uncultivated. The distance between adjacent sites varied between 10 and $25 \mathrm{~km}$. At each site, soil specimens were taken from the waterside and $100 \mathrm{~m}$ inland at 10 different locations spaced 100-200 m apart.

A specimen of $c .100 \mathrm{~g}$ was sampled from the soil at a depth of $15-20 \mathrm{~cm}$.

\section{Isolation of neurotoxigenic C. butyricum}

The presence of botulinum toxin-producing organisms in soil was demonstrated by the method described by Yamakawa et al. [7]. Ten 1-g portions of each soil specimen were inoculated into 10 tubes containing a chopped meat-glucose (CMG) medium [8] and incubated anaerobically at $30^{\circ} \mathrm{C}$ for 5 days. The culture was frozen, thawed, and then each culture supernate was examined in the mouse lethality tests for type $E$ botulinum toxicity with and without trypsinisation. For trypsinisation, the supernates were mixed with equal volumes of trypsin ( 1 in 250; Difco Laboratories, Detroit, MI, USA) $0.5 \%$ in $0.05 \mathrm{M}$ phosphate buffer at $\mathrm{pH} 6.5$, and incubated for $30 \mathrm{~min}$ at $37^{\circ} \mathrm{C}$. Each culture in which type $\mathrm{E}$ botulinum toxicity was demonstrated was then spread on 10 plates of egg yolk-containing trypticase peptone-yeast extract-glucose (egg yolkTYG) agar - egg yolk 5\%; trypticase peptone (Beckton Dickinson Microbiology Systems, Cockeysville, MD, USA) $20 \mathrm{~g} / \mathrm{L}$; yeast extract $5 \mathrm{~g} / \mathrm{L}$; glucose $5 \mathrm{~g} / \mathrm{L} ; \mathrm{NaCl}$ $0.9 \mathrm{~g} / \mathrm{L}$; agar $20 \mathrm{~g} / \mathrm{L} ; \mathrm{pH} 7.2$ - and incubated anaerobically for 2 days. Between 12 and 20 lipaseand lecithinase-negative colonies were picked from each culture, inoculated into CMG medium and incubated anaerobically at $30^{\circ} \mathrm{C}$ for 5 days. The supernate of the culture was examined for toxicity by the mouse lethality tests. When toxic activity was detected, the culture was checked for purity by re-cultivation on egg yolk-TYG agar and subjected to tests for biochemical properties and polymerase chain reaction (PCR) for detection of the type $\mathrm{E}$ botulinum toxin gene.

\section{Biochemical properties of the isolates}

Tests for biochemical properties were performed according to the procedures described by Nakamura et al. [9]. Gas chromatographic analysis of fermentation products in PYG medium was performed according to the method of Holdeman et al. [8].

\section{$P C R$}

Detection of the type E botulinum toxin gene by PCR was performed with the primer set of E1 (5'TATATATTAAACCAGGCGG-3') and E2 (5'-TAGAGAAATATTGGAACTG-3') as described by Szabo $e t$ al. [10]. A $100-\mu 1$ volume of bacterial culture in Brain Heart Infusion Broth (Beckton Dickinson Microbiology Systems) was boiled for $5 \mathrm{~min}$, centrifuged and then the supernate was used as a PCR template. The amplification was accomplished in $50-\mu 1$ volumes containing $2 \mu \mathrm{l}$ of the boiled cell lysate, $50 \mathrm{pmol}$ of each primer, $0.2 \mathrm{mM}$ each $\mathrm{dNTP}, 1.5 \mathrm{mM} \mathrm{MgCl}_{2}$, $50 \mathrm{mM} \mathrm{KCl}, 10 \mathrm{mM}$ Tris-HCl, $\mathrm{pH} 8.3$, gelatin $0.001 \%$ $\mathrm{w} / \mathrm{v}$ and 2.5 units of AmpliTaq DNA polymerase (Perkin-Elmer, Foster City, CA, USA) with an Omn-E thermal cycler (Hybaid, Middlesex). The amplification conditions were 30 cycles of $94^{\circ} \mathrm{C}$ for $1 \mathrm{~min}, 45^{\circ} \mathrm{C}$ for $1 \mathrm{~min}$ and $72^{\circ} \mathrm{C}$ for $1 \mathrm{~min}$. PCR products were separated on agarose gel and visualised with ethidium bromide under UV light.

\section{Results}

\section{Isolation of neurotoxigenic organisms}

Type $\mathrm{E}$ botulinum toxicity was found in the soil cultures at all sites examined; toxicity-positive soil specimens were found in one to nine of 10 soil 
specimens at each site (Table 1). Numbers of the toxicity-positive cultures were one to four of 10 cultures in each toxicity-positive specimen; 46 in total. The cultures exhibited one to 16 or more minimal lethal doses (MLD)/ml of type $\mathrm{E}$ toxicity, which increased to $20-\geqslant 100 \mathrm{MLD} / \mathrm{ml}$ after treatment with trypsin. All 46 toxicity-positive culture tubes were investigated to isolate the neurotoxigenic organism. Type E toxicity-positive organisms were isolated from seven of these cultures. The seven cultures were from seven different soil specimens, and in summary, seven isolates were obtained from seven different soil specimens which were collected from four different sites: Guanyun and the lakeside sites A, C and D.

\section{Biochemical properties}

The biochemical properties of all seven isolates plus the two stock strains were examined. Except for inulin fermentation and starch hydrolysis, no difference was found among the nine isolates in the properties tested; inulin fermentation was positive in the isolates from soil in the Weishan lake area, and starch hydrolysis in two of five isolates from soil in the Weishan lake area and two stock strains (Table 2).

Gas chromatographic analysis of fermentation products showed major peaks for acetic and butyric acids and minor peaks for lactic and succinic acids; no difference was found in fermentation products.

Type $E$ toxicity of the culture supernates of the isolates were $10^{2}-10^{3} \mathrm{MLD} / \mathrm{ml}$, which increased $10-$ 1000-fold after treatment with trypsin.

On the basis of these findings, the isolates were identified as neurotoxigenic $C$. butyricum.

\section{Detection of type $E$ botulinum toxin gene}

PCR with type $\mathrm{E}$ botulinum toxin gene-specific primers was performed to detect the type $\mathrm{E}$ botulinum toxin

Table 1. Isolation of neurotoxigenic C. butyricum from soil specimens

\begin{tabular}{lcccc}
\hline & Soil & $\begin{array}{c}\text { Type } \mathrm{E} \\
\text { botulinum toxicity- } \\
\text { positive specimens }\end{array}$ & $\begin{array}{c}\text { Type E } \\
\text { botulinum toxicity- } \\
\text { positive cultures* }\end{array}$ & $\begin{array}{c}\text { Neurotoxigenic } \\
\text { C. butyricum isolation } \\
\text { positive specimens }\end{array}$ \\
\hline Guanyun & specimens examined & 4 & 5 & 2 \\
Weishan lake & 10 & 4 & 5 & 3 \\
A & 10 & 4 & 1 & 0 \\
B & 10 & 1 & 8 & 1 \\
C & 10 & 3 & 25 & 1 \\
D & 10 & 9 & 2 & 0 \\
E & 10 & 1 & 5 & \\
\hline
\end{tabular}

${ }^{*}$ A 10 -g specimen was equally divided into 10 parts and examined for toxicity of the cultures. One to four of the 10 cultures were positive in each type $\mathrm{E}$ botulinum toxicity-positive specimen.

${ }^{\dagger}$ Each strain was isolated from a different soil specimen and examined further for biochemical properties.

Table 2. Properties of neurotoxigenic C. butyricum isolates

\begin{tabular}{|c|c|c|c|c|c|}
\hline \multirow[b]{2}{*}{ Property } & \multicolumn{2}{|c|}{ Isolate from soil in } & \multicolumn{2}{|c|}{$\begin{array}{l}\text { Collection strains } \\
\text { from botulism cases }\end{array}$} & \multirow[b]{2}{*}{$\begin{array}{l}\text { C. butyricum } \\
\text { NCIB } 7423^{*}\end{array}$} \\
\hline & $\begin{array}{l}\text { Guanyun } \\
\text { (2) }\end{array}$ & $\begin{array}{c}\text { Weishan lake } \\
\text { (5) }\end{array}$ & LCL 063 & LCL 095 & \\
\hline Lecithinase & - & - & - & - & - \\
\hline Lipase & - & - & - & - & - \\
\hline Liquefaction of gelatin $(2 \%, 10 \%)$ & - & - & - & - & - \\
\hline Digestion of casein & - & - & - & - & - \\
\hline Digestion of meat & - & - & - & - & - \\
\hline Milk reaction & $\mathrm{c}$ & $\mathrm{c}$ & $\mathrm{c}$ & $\mathrm{c}$ & $\mathrm{c}$ \\
\hline Indole production & - & - & - & - & - \\
\hline Nitrate reduction & - & - & - & - & - \\
\hline Acid production ${ }^{\dagger}$ & + & + & + & + & + \\
\hline Inulin & - & + & - & - & + \\
\hline $\begin{array}{l}\text { Adonitol, arabinose, dulcitol, erythritol, inositol, } \\
\text { mannitol, rhamnose, sorbitol, sorbose }\end{array}$ & - & - & - & - & - \\
\hline Aesculin hydrolysis & + & + & + & + & + \\
\hline Starch hydrolysis & - & - or + & + & + & + \\
\hline Sodium chloride tolerance $(\%)$ & 2 & 2 & 2 & 2 & 2 \\
\hline Product from $\mathrm{PYG}$ & $\mathrm{ABls}^{\S}$ & ABls & $\mathrm{ABls}$ & $\mathrm{ABls}$ & $\mathrm{ABls}$ \\
\hline Toxicity (MLD/ml) & $10^{5}-10^{6}$ & $10^{3}-10^{5}$ & $10^{5}$ & $10^{5}$ & No \\
\hline
\end{tabular}

c, curd.

${ }^{*}$ Refer to reference 6 .

†From: aesculin, amygdalin, arbutin, cellobiose, dextrin, fructose, galactose, glucose, glycogen, lactose, maltose, mannose, raffinose, ribose, salicin, starch, sucrose, trehalose, xylose.

$\S_{\mathrm{A}}$, acetic acid; B, n-butyric acid; 1 , lactic acid; s, succinic acid. Capital letters, $\geqslant 10 \mathrm{mM}$; small letters, $<10 \mathrm{mM}$.

After trypsin treatment. 

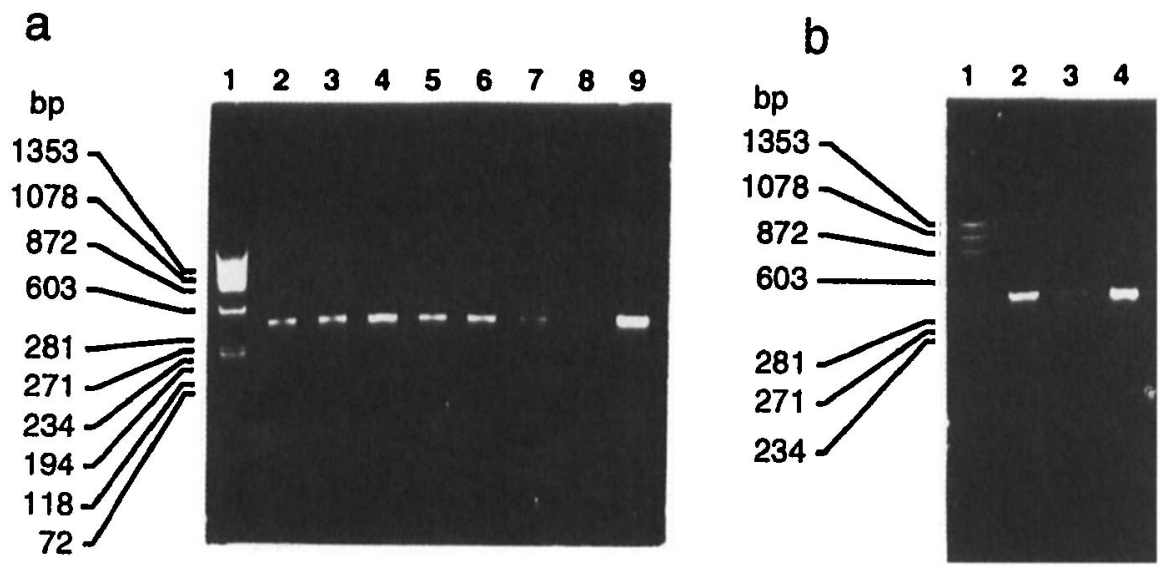

Fig. 2. Agarose gel electrophoresis of PCR products amplified with the primers specific for the type $\mathrm{E}$ botulinum toxin gene. (a) Lane 1, Hae III-digested phi-X $174 \mathrm{DNA} ; \mathbf{2 - 4}$, isolates from site A; 5, an isolate from site C; 6, an isolate from site D; 7 and 8, isolates from Guanyun; 9, neurotoxigenic C. butyricum BL 6340. (b) Lane 1, Hae III-digested phi-X 174 DNA; 2, an isolate from the botulism outbreak at Jining in $1973 ; 3$, an isolate from the botulism outbreak at Peixian in $1983 ; 4$, neurotoxigenic C. butyricum BL 6340.

gene. A product of the expected size (446 bp) was amplified from the soil isolates and from $C$. butyricum BL 6340 (Fig. 2).

\section{Discussion}

A recent study isolated neurotoxigenic $C$. butyricum from the food implicated in an outbreak of type $E$ botulism [5]. This prompted the examination of soil from the area around the patients' house (Guanyun site) for neurotoxigenic $C$. butyricum and the reexamination of stock strains from the Lanzhou Institute, which had been isolated from clinical samples from patients with type $\mathrm{E}$ botulism in China. This study showed retrospectively that two type $\mathrm{E}$ botulinum toxin-producing strains isolated previously in the area near Weishan lake had the properties of $C$. butyricum. On the basis of these findings, lakeside soil from Weishan lake was examined for neurotoxigenic $C$. butyricum.

Corresponding to the principle that toxin types of food-borne botulism caused by homemade foods are usually consistent with those of $C$. botulinum found locally in soil, neurotoxigenic $C$. butyricum was isolated from soil from both areas, i.e., Guanyun and Weishan lake. The biochemical properties of the isolates from soil at the Guanyun site agreed completely with those of an isolate from the food implicated as a source of botulism in patients living near that site [5], suggesting that the food may have been contaminated with the organism from soil in the environment of the patients involved in that outbreak. In the isolates from soil of the lakeside of the Weishan lake area, inulin fermentation was positive, differing from the results with the stock strains which had been isolated from samples from botulism patients at Jining and Peixian, which are both $c .10 \mathrm{~km}$ from Weishan lake. There could be at least two different clones of neurotoxigenic $C$. butyricum in this area. In both the Guanyun and Weishan lake areas, genetic comparison of environmental and clinical isolates of C. butyricum remains to be done.

Neurotoxigenic $C$. butyricum was first isolated in two cases of infant botulism in Rome, 1986 [2]. Since then the properties of a neurotoxin from the organism have been extensively studied physicochemically, immunologically and genetically [11-13]. However, distribution of this organism in soil has not been reported. Creti et al. [14] attempted to detect neurotoxigenic $C$. butyricum in soil in the vicinity of Rome after the outbreak, but were unable to do so. The present study revealed for the first time that neurotoxigenic $C$. butyricum is distributed in soil in the same manner as C. botulinum types A, B, E, F and G. In China, as well as in other countries, studies on the distribution of C. botulinum in soil have been performed in most cases by detecting botulinum toxin in the soil cultures, without isolation of $C$. botulinum [15]. The major reason why isolation of the bacterium has not been done extensively seems to be due to the general concept that $C$. botulinum organisms corresponding to a toxin type found in soil culture must exist in the soil. We have been studying the distribution of $C$. botulinum in soil since 1974 and have often encountered cases where $C$. botulinum type E could not be isolated from soil cultures with type E botulinum toxicity, although lipase-positive colonies were examined. The present study indicates that there are neurotoxigenic $C$. butyricum in the area where soil cultures show type E botulinum toxicity, but $C$. botulinum type E lipasepositive colonies were not isolated.

\section{References}

1. Smith, LDS, Williams BL. Clostridium botulinum. In: The pathogenic anaerobic bacteria, 3rd edn. Springfield, IL, Charles C. Thomas. 1984: 148-163. 
2. Aureli P, Fenicia L, Pasolini B, Gianfranceschi M, McCroskey LM, Hatheway CL. Two cases of type $\mathrm{E}$ infant botulism caused by neurotoxigenic Clostridium butyricum in Italy. $J$ Infect Dis 1986; 154: 207-211.

3. McCroskey LM, Hatheway CL, Fenicia L, Pasolini B, Aureli P. Characterization of an organism that produces type $\mathrm{E}$ botulinal toxin but which resembles Clostridium butyricum from the feces of an infant with type E botulism. J Clin Microbiol 1986; 23: $201-202$.

4. Hall JD, McCroskey LM, Pincomb BJ, Hatheway CL. Isolation of an organism resembling Clostridium barati which produces type $\mathrm{F}$ botulinal toxin from an infant with botulism. $J$ Clin Microbiol 1985; 21: 654-655.

5. Meng X, Karasawa $\mathrm{T}$, Zou $\mathrm{K}$ et al. Characterization of a neurotoxigenic Clostridium butyricum strain isolated from the food implicated in an outbreak of food-borne type E botulism. $J$ Clin Microbiol 1997; 35: 2160-2162.

6. Zhao W, Lin H, Qian L, Gu X, Dong S, Wang L. Study on an outbreak of type E botulism. Chin J Health Lab Technol 1995; 5: $62-63$ (in Chinese).

7. Yamakawa K, Nakamura S. Prevalence of Clostridium botulinum type $\mathrm{E}$ and coexistence of $C$. botulinum nonproteolytic type B in the river soil of Japan. Microbiol Immunol 1992; 36: 583-591.

8. Holdeman LV, Cato EP, Moore WEC. Anaerobe laboratory manual, 4th edn. Blacksburg, VA, Virginia Polytechnic Institute and State University, 1977.
9. Nakamura S, Shimamura T, Hayase M, Nishida S. Numerical taxonomy of saccharolytic clostridia, particularly Clostridium perfringens-like strains: descriptions of Clostridium absonun $\mathrm{sp}$. n. and Clostridium paraperfringens. Int J Syst Bacteriol 1977; 23: 419-429.

10. Szabo EA, Pemberton JM, Desmarchelier PM. Detection of the genes encoding botulinum neurotoxin types $\mathrm{A}$ to $\mathrm{E}$ by the polymerase chain reaction. Appl Environ Microbiol 1993; 59: 3011-3020.

11. Giménez JA, Sugiyama H. Comparison of toxins of Clostridium butyricum and Clostridium botulinum type E. Infect Immun 1988; 56: 926-929.

12. Kozaki S, Onimaru J, Kamata Y, Sakaguchi G. Immunological characterization of Clostridium butyricum neurotoxin and its trypsin-induced fragment by use of monoclonal antibodies against Clostridium botulinum type E neurotoxin. Infect Immun 1991; 59: 457-459.

13. Poulet S, Hauser D, Quanz M, Niemann H, Popoff MR. Sequences of the botulinal neurotoxin $E$ derived from Clostridium botulinum type E (strain Beluga) and Clostridium butyricum (strains ATCC 43181 and ATCC 43755). Biochem Biophys Res Commun 1992; 183: 107-113.

14. Creti R, Fenicia L, Aureli P. Occurrence of Clostridium botulinum in the soil of the vicinity of Rome. Curr Microbiol 1990; 20: 317-321.

15. Gao QY, Huang YF, Wu JG, Liu HD, Xia HQ. A review of botulism in China. Biomed Environ Sci 1990; 3: 326-336. 